\title{
Cellular and molecular aspects of drug-induced liver toxicity: recent prominent mechanisms
}

\begin{abstract}
Despite recent advances in drug development technologies, some drugs continue to be withdrawn from the market because of their late hepatotoxic effects. Drugs can cause liver toxicity through several molecular mechanisms, most of which need to be clarified by further research. Newly found drug-induced liver injury (DILI)-mechanisms like endoplasmic reticulum stress should also be considered while evaluating drug toxicity. New drugs launched in the market should be tested particularly for hepatotoxicity by using hepatic cell cultures and animal models continuously. Future research should target patients in the post-marketing phase in order to provide more evidence grounds to the clinical safety of potential drugs of high propensity of DILI. This review aims to draw the attention to the issue of cellular and molecular aspects of liver toxicity induced by drugs and address DILI as a potential clinical problem that needs further investigation.
\end{abstract}

Keywords:drug-induced liver injury, hepatotoxic drugs, molecular mechanisms, endoplasmic reticulum stress

\author{
Volume I Issue 5 - 2015 \\ Pinar Erkekoglu,' Asim Elnour, ${ }^{2}$ Belma Kocer- \\ Gümüșel,' Akshaya Srikanth Bhagavathula, ${ }^{3}$ \\ Abdulla Shehab ${ }^{4}$ \\ 'Department of Toxicology, Faculty of Pharmacy, Hacettepe \\ University, Turkey \\ ${ }^{2}$ Department of Pharmacology, College of Medicine and Health \\ Sciences, UAE University, UAE \\ ${ }^{3}$ Department of Clinical Pharmacy, College of Medicine and \\ Health Sciences, University of Gondar, Ethiopia \\ ${ }^{4}$ Department of Internal Medicine, College of Medicine and \\ Health Sciences, UAE University, UAE
}

Correspondence: Pinar Erkekoglu, Department ofToxicology, Faculty of Pharmacy, Hacettepe University, Turkey, Tel 00903 |23052 I78, Fax 00903 I23। I 4777. Email erkekp@yahoo.com

Received: November 18, 2015 | Published: November 27, 2015
Abbreviations: ALT, alanine transaminase; AP, alkaline phosphatase; CYP450, cytochrome p450; DILI, drug induced liver injury; GSH, glutathione; HIV, human immunodeficiency virus; LASH, lactic acidosis, acute microvesicular steatosis and hepatic dysfunction; NAFLD, non alcoholic fatty liver disease; PARP-1, poly (adenosine diphosphate-adp-ribose) polymerase; SJS, stevens johnson syndrome; TEN, toxic epidermal necrolysis; UL, upper limit

\section{Introduction}

The liver is a vital organ for all higher organisms. It has a wide range of functions, ranging from the biotransformation (particularly detoxification) of various chemicals to protein synthesis (i.e., plasma proteins); from glycogen storage and lipogenesis to bile production. ${ }^{1}$ Drug-induced liver diseases are particularly caused by prescribed medications, as well as over-the-counter medications, vitamins, hormones, herbs, illicit ("recreational") drugs, and environmental toxins. While the liver is exposed to many xenobiotics (including several drugs), its injury is inevitable when successful regeneration is not possible. Drugs continue to be withdrawn from the market because of late discovery of their hepatotoxic effects.

This review aims to draw the attention to the issue of "druginduced liver injury (DILI)" as a potential clinical problem that needs further exploration. In this framework, the newly discovered underlying mechanisms of DILI are being addressed and literature is reviewed through relevant articles that address DILI.

\section{Epidemiology of drug-induced liver injury (DILI)}

The estimated annual incidence of DILI is $10-15$ per 10.000 to 100.000 persons who are taking prescripted medications. ${ }^{2}$ In addition, DILI accounts for approximately $10 \%$ of acute hepatitis cases annually and it is the most common cause of acute liver failure in the United States. ${ }^{3-5}$ DILI is also one of the most common causes of medication withdrawal from the market. ${ }^{4,6,7}$ DILI may not be detected prior to drug approval as Phase III trials are performed on $<3000$ people. Therefore, cases of DILI with an incidence of 1 in 10,000 may be missed and this will determine the future of the drug on the market. It has been estimated that in a clinical trial, for every 10 cases of alanine amino transferase elevation ( $>10$ times the upper limit of normal), there will be one case of severe liver injury. ${ }^{8,9}$ Different factors such as gender, age, ethnicity/race, nutritional status (malnutrition, high fat consumption), other pathological conditions (renal dysfunction, liver disease, obesity, diabetes mellitus, infections, inflammatory diseases), addictions (high alcohol consumption, smoking) and pregnancy might also affect the emergence of DILI. Besides, drugrelated factors (duration, dosage, dosage form, usage of other drugs and drug interactions) and metabolic and enzymatic profile (genetic polymorphisms) can also be the underlying factor for DILI. The pathophysiology of DILI varies depending on the drug and, in many cases, is not entirely understood. DILI mechanisms include covalent binding of the drug to cellular proteins resulting in immune injury, direct cytotoxicity, inhibition of cell metabolic pathways, blockage of cellular transport pumps, induction of apoptosis, and interference with mitochondrial function. ${ }^{10}$

\section{The mechanism of drug-induced liver injury}

\section{Cytotoxicity}

Cytotoxicity is one of the main mechanisms that may lead to acute or chronic liver injury. Cytotoxicity can be caused by several factors, including oxidative stress. Changes in the expression of antioxidant enzymes and in the synthesis or degradation of cellular thiols, increases in lipid peroxidation and protein oxidation are good markers of cellular oxidation. ${ }^{11,12}$ On the other hand, disruption of thiol synthesis 
or oxidation of cellular thiols, particularly high oxidation of glutathione (GSH), may also be an underlying factor for cellular toxicity. ${ }^{11,12}$ Cytotoxicity may be followed by cell death, by either apoptosis or necrosis.

\section{Apoptosis}

Apoptosis is a form of programmed cell death and activation of caspase pathways (intrinsic or extrinsic). Chemicals and drugs usually activate extrinsic caspase pathway, leading to increase in caspase 3 and later caspase 8 activities, and to poly (adenosine diphosphate-ADP-ribose) polymerase (PARP) cleavage. It has been suggested that the mode of death for acetaminophen's liver toxicity is caspase-independent apoptosis, initiated by activation of PARP$1 .^{13-15}$ The widely used antidepressant, sertraline was shown to cause to apoptosis and mitochondrial dysfunction in primary rat hepatocytes and human HepG2 cells. ${ }^{16}$

\section{Necrosis}

Necrosis, on the other hand, is a form of cell injury that results in the premature death of cells in living tissue by autolysis. On a cellular basis, it is suggested that hepatic necrosis is the result of overwhelming or deregulated apoptosis. ${ }^{17}$ Besides, exaggerated mitochondrial dysfunction or lysosomal permeabilization can result in cell death (by either apoptosis or necrosis). ${ }^{17,18}$ However, regeneration of hepatocytes lost by necrotic and apoptotic cell death may mask detection of DILI. ${ }^{19,20}$

Zonal necrosis is the most common type of necrosis that is induced by drugs. In this specific pathological condition, the injury largely remains limited to a particular zone of a liver lobule leading to a very high level of ALT, severe disturbance of liver function and finally to acute liver failure. ${ }^{21}$ Acetaminophen, valproic acid and carbon tetrachloride can lead to zonal necrosis if taken in high doses. ${ }^{22}$

\section{Endoplasmic reticulum (ER) stress}

The endoplasmic reticulum (ER) is an organelle in the eukaryotic cells. It plays a vital role in many cellular processes such as protein synthesis, folding, assembly, trafficking and post-modulation. Besides, it provides quality control of both secretory and membrane proteins, lipid synthesis, and regulation of intracellular calcium hemostasis. ER stress is an exciting area of research in liver injury, although available data mostly comes from other experimental systems. The stressed ER exhibits an imbalance between unfolded proteins and mature proteins, activating a series of compensatory responses, collectively termed as "unfolded protein response". ${ }^{23-25}$ Three ER membrane-localized proteins are considered as 'sensors' of ER stress: inositol-requiring enzyme 1 (IRE1), activating transcription factor 6 (ATF6), and protein kinase RNA-activated (PKR)-like ER kinase (PERK) ${ }^{26}$ ER stress may occur before apoptotic cell death and glutathione (GSH) depletion. Excessive and prolonged ER stress finally leads to apoptosis or necrotic cell death. ${ }^{27}$ However, ER stress may also occur after a cascade of events, such as GSH depletion and oxidative stress. ${ }^{28}$

A wide range of human diseases, have been associated with ER stress. These diseases include, but are not limited to, neurological (Alzheimer's disease, Parkinson's disease), kidney, lung, and cardiovascular diseases, diabetes, obesity/metabolic syndrome, inflammatory diseases and cancer. ${ }^{23}$ In addition, environmental toxicants as well as drugs were related to ER stress in the last reports. In liver, ER stress can be induced by several factors, including drugs,
UV radiation, and insulin resistance. ER stress can regulate both the intrinsic and extrinsic cell death machinery. Studies in the last decade have shown that non-steroidal anti-inflammatory drugs (NSAIDs, such as indomethacin, diclofenac), benzodiazepines, lithium and sodium valproate were shown to induce ER stress both in vitro or in vivo. ${ }^{25}$ The HIV-protease inhibitors, atazanavir and ritonavir were shown to induce both ER stress and apoptosis. Moreover, the mechanisms involved in the liver toxicity of anti-diabetic drug troglitazone, include mitochondrial dysfunction, apoptosis, disruption of calcium hemostasis, oxidative stress, and ER stress. ${ }^{23}$ Although the most important mechanism for sertaline-induced liver toxicity is mitochondrial dysfunction and apoptosis, ER stress was found to contribute to its liver toxicity. ${ }^{29}$ Interestingly, certain antioxidants (butylated hydroxyanisole (BHA), TM2002, and Baicalein) were also shown to cause ER stress in liver. ${ }^{30}$

\section{Mitochondrial dysfunction}

Mitochondria are the key organelles for intracellular energy generation. They are involved in several metabolic pathways including cellular respiration. These organelles are highly organized, have high metabolic capacity and act through various signaling networks. Mitochondria are directly or indirectly involved in the activation of intracellular stress cascades, apoptosis, and necrosis or death receptor-mediated pathways. ${ }^{31}$ High intracellular ROS levels accompanied by GSH depletion, lipid peroxidation, protein oxidation/alkylation and respiratory complex changes are associated with mitochondrial dysfunction. These events may be the critical underlying factors for many types of chronic liver diseases and for DILI. ${ }^{32}$ Moreover, the formation of reactive metabolites, after the biotransformation of drug with Phase I or Phase II enzymes, can trigger hepatitis through direct toxicity or immune reactions. These reactions might also cause mitochondrial membrane disruption. ${ }^{33}$ On the other hand, parent drug can also lead to mitochondrial dysfunction through different mechanisms: ${ }^{33-35}$

\section{i. Direct inhibition of mitochondrial functions}

ii. Inhibition of mitochondrial DNA synthesis (acetaminophen, alcohol, ganciclovir, tamoxifen, troglitazone, zidovudine)

iii. Impairment of mitochondrial DNA synthesis (fialuridine, ciprofloxacin, nalidixic acid)

iv. Disruption in mitochondrial DNA protein synthesis (chloramphenicol, erythromycin, thiamphenicol)

v. Decrease mitochondrial transcripts (interferon- $\alpha$ )

vi. Impairment in oxidative phosphorylation and secondarily inhibition $\beta$-oxidation leading to steatosis, cell death, mostly necrosis (e.g. amineptine, amiodarone, aspirin, chloroquine, perhexiline, tetracyclines and tianeptine)

vii. Disruption in pyruvate catabolism, leading to lactic acidosis

viii. Decreased gluconeogenesis

ix. Accumulation of free fatty acids and lipid peroxidation products, leading to the impairment of energy production

\section{Types of drug induced liver toxicity}

Several different medications have been linked to the development of DILI. Drug induced liver injury (DILI) can be predictable (occurs shortly after exposure, dose-related) or unpredictable (develops after 
a period of latency, dose-independent). Subclinical DILI may be underreported. ${ }^{36}$

DILI can be classified as follows:

Idiosyncratic DILI: DILI is mostly observed as an idiosyncratic reaction that may or may not be dose-related. It is unpredictable, and usually occurs after a latent period. ${ }^{37}$ It is a rare cause of severe liver disease.

Intrinsic DILI: It is a dose dependent (i.e. acetaminophen-induced) and predictable event. There is a threshold dose for each chemical, above which individuals respond with liver toxicity that becomes more severe with increasing dose. ${ }^{38}$

Chronic DILI: If there is chronic exposure to a hepatotoxic drug, DILI proceeds. There becomes a failure of return of liver enzymes or bilirubin to pre-DILI baseline. Later, other signs or symptoms of ongoing liver disease (e.g., Ascites, encephalopathy, portal hypertension, and coagulopathy) may be observed in 6 months. As liver has a high reserve of albumin, a decrease of the content of albumin tends to be associated with chronic DILI. Chronic DILI predisposes to acute liver failure, acute jaundice, cirrhosis and finally death. ${ }^{39}$

Concerning the type of liver damage, DILI can cause the damages listed below: $:^{40-42}$

\section{Hepatocellular damage}

This type of injury can result from drugs such as acetaminophen and isoniazid.

Symptoms:

a. Malaise

b. Right upper quadrant abdominal pain

c. Marked elevation in amino transferase levels (ALT, AST, or both)

d. Hyperbilirubinemia (with jaundice, impaired hepatic synthesis, and encephalopathy)

\section{Cholestatic damage}

Substances known to lead to this type of injury include amoxicillin/ clavulanate and chlorpromazine. This type of injury is usually less serious than severe hepatocellular damage.

\section{Symptoms:}

a. Development of pruritus and jaundice

b. Marked elevation of serum alkaline phosphatase (AP) levels.

c. Vanishing bile duct syndrome (rarely).

Mixed damage: Drugs such as phenytoin can cause this type of injury. Neither aminotransferase nor AP elevations are clearly predominant. Symptoms may also be mixed.

\section{Clinical significance of drug-induced liver injury}

Many drugs, medicinal herbs, plants, and nutritional supplements can induce liver injury in acute or chronic use..$^{43}$ The term DILI may be used to express clinically significant liver injury or asymptomatic liver injury. The most specific predictor for a drug's potential for severe hepatotoxicity, however, is the aminotransferase elevations, which can be accompanied by increase in serum total bilirubin (TBL). ${ }^{30}$ Hyperbilirubinemia leads to hepatocellular jaundice and, according to Hy's law, is associated with mortality rates as high as $50 \%$. Drugs most commonly cause asymptomatic elevation of hepatic enzymes (aspartate amino transferase (AST), alanine amino transferase (ALT), which is more specific, and AP). Although the finding of a higher rate of such elevations in subjects using drugs than in a control group is a sensitive signal of a potential to cause severe DILI, it is not specific signal. A more specific signal of such potential is a higher rate of more marked peak ALT elevations (10x, 15x upper limit of normal-ULN), with cases of increases to $>1,000 \mathrm{U} / \mathrm{L}$ causing more concerns. ${ }^{44}$

Increased plasma prothrombin (PT) time, or its international normalized ratio (INR) because of reduced hepatic production of Vitamin K-dependent clotting factors, is another potentially useful measure of liver function that might suggest DILI. ${ }^{44}$ However, clinically significant liver injury (e.g. jaundice, abdominal pain, or pruritus) or impaired liver function, resulting in deficient protein synthesis (with prolonged PT or with hypo albuminemia) is rare. DILI includes injury caused by medicinal herbs, plants, and nutritional supplements as well as drugs. ${ }^{43,44}$

\section{Management of drug-induced liver injury}

Early drug withdrawal usually results in recovery. In severe cases, consultation with a specialist is indicated, especially if patients have hepatocellular jaundice and impaired liver function, because liver transplantation may be required. ${ }^{45,46}$ Antidotes for DILI are available for only a few hepatotoxins; such as $N$-acetylcysteine for acetaminophen. ${ }^{47}$

\section{Prevention of drug-induced liver injury}

The preventive strategies to prevent DILI are always taken seriously and initiated during the drug development process; however, such efforts may not ensure the drug safety in Phase IV (postmarketing) due to a larger population exposure. The post-marketing surveillance, now increasingly controlled by Food and Drug Agency (FDA) in United States, may enhance the awareness to potentially hepatotoxic drugs. ${ }^{44}$ Many countries, including Turkey, have their own pharmacovigilance systems and these systems provide a high rate of observation of drug-related injuries, including DILI.

In United States, the National Institute of Diabetes and Digestive and Kidney Diseases (NIDDK) has established the Drug-Induced Liver Injury Network (DILIN) to collect and analyze cases of severe liver injury induced by prescribed drugs, over-the-counter (OTC) drugs, and alternative medicines, such as herbal products and dietary supplements. ${ }^{48}$ Besides, The National Institutes of Health (NIH) has created a database named "LIVERTOX" for drugs that may lead to the development of DILI. It has been suggested that the use of pharmacogenomics in the near future may allow tailoring of drug use and avoidance of potential toxicities in susceptible individuals, like children, elderly and pregnant. ${ }^{49}$

\section{Discussion}

Drugs cause liver injury by many different mechanisms. DILI can be predictable, dose-related or unpredictable, unrelated to the dose of the drug on question. DILI can exist as hepatocellular damage, cholestatic damage (usually less serious than hepatocellular), or mixed damage. ${ }^{50}$ 
The risk factors associated with DILI include age ( $<18$ or $>65$ years), obesity, pregnancy, concomitant alcohol consumption, and certain genetic polymorphisms (such as cytochrome $\mathrm{P} 450$ polymorphisms). It has been suggested that susceptible populations should not be subjective to re-challenge with drugs suspected of causing DILI. ${ }^{49,50-53}$ These injuries resemble almost all known liver diseases and there are no pathognomonic findings, even upon liver biopsy, that clarify the diagnosis of DILI. Therefore, when the clinician suspects of DILI, it is essential to gather additional clinical and laboratory information necessary for differential diagnosis of the cause. The most important key point is to exclude other causes of liver disease (existence of acute/chronic hepatitis, nonalcoholic steatohepatitis [NASH], biliary tract diseases (stones, sand), obesity/metabolic syndrome, circulatory problems, congestive heart failure, concomitant exposure to other hepatotoxic drugs, alcohol or hepatotoxins). ${ }^{51-53}$ Some of the drugs that induce DILI and the mechanisms underlying their toxicity are summarized in Table $1 .^{54-78}$

There are several molecular mechanisms induced by drugs that can be suggested for liver toxicity, most of which need to be clarified by further research. New drugs launched in the market should be tested especially for hepatotoxicity by using hepatic cell cultures and animal models. The pharmaceutical companies and researchers should particularly focus on late hepatotoxicity as it is sneaky and most dangerous effect, which can lead to high morbidity. Moreover, newly found toxicity mechanisms like ER stress should also be considered while evaluating drug toxicity. Future research should target patients in the post-marketing phase in order to provide more evidence grounds to the clinical safety of potential drugs of high propensity to DILI.

\section{Acknowledgements}

None.

\section{Conflict of interest}

The author declares no conflict of interest

\section{References}

1. Jaeschke H, Gores GJ, Cederbaum AI, et al. Mechanisms of hepatotoxicity. Toxicol Sci. 2002;65(2):166-176.

2. Sgro C, Clinard F, Ouazir K. Incidence of drug-induced hepatic injuries:a French population-based study. Hepatology. 2002;36(2):451455.

3. Zimmerman HJ. Drug-induced liver disease. Clin Liver Dis. 2000;4:7396.

4. Ostapowicz G, Fontana RJ, Schiodt FV. Results of a prospective study of acute liver failure at 17 tertiary care centers in the United States. Ann Intern Med. 2002;137(12):947-954.

5. Larson AM, Polson J, Fontana RJ. Acetaminophen-induced acute liver failure:results of a United States multicenter, prospective study. Hepatology. 2005;42(6):1364-1372

6. Regev A. Drug-induced liver injury and drug development: industry perspective. Semin Liver Dis. 2014;34(2):227-239.

7. Xu JJ, Diaz D, O’Brien PJ. Applications of cytotoxicity assays and pre-lethal mechanistic assays for assessment of human hepatotoxicity potential. Chem Biol Interact. 2004;150(1):115-128.

8. Maddur H, Chalasani N. Idiosyncratic drug-induced liver injury:a clinical update. Curr Gastroenterol Rep. 2011;13(1):65-71.
9. Lewis JH. 'Hy's law,' the 'Rezulin Rule,' and other predictors of severe drug-induced hepatotoxicity:putting risk-benefit into perspective. Pharmacoepidemiol Drug Saf. 2006;15(4):221-229.

10. Choi JH, Choi AY, Yoon H. Baicalein protects HT22 murine hippocampal neuronal cells against endoplasmic reticulumstress-induced apoptosis through inhibition of reactiveoxygen species production and $\mathrm{CHOP}$ induction. Exp Mol Med. 2010;42(12):811-822.

11. Halliwell B. Cell culture, oxidative stress, and antioxidants:avoiding pitfalls. Biomed J. 2014;37(3):99-105

12. Halliwell B. The antioxidant paradox:less paradoxical now? Br J Clin Pharmacol. 2013;75(3):637-44.

13. Estaquier J, Vallette F, Vayssiere JL, et al. The mitochondrial pathway of apoptosis. Adv in Mitochond Med. 2012;942:157-183.

14. Nagy G, Szarka A, Lotz G. BGP-15 inhibits caspase-independent programmed cell death in acetaminophen-induced liver injury. Toxicol Appl Pharmacol. 2010;243(1):96-103.

15. Hwang HJ, Kwon MJ, Nam TJ. Chemoprotective effect of insulin-like growth factor I against acetaminophen-induced cell death in Chang liver cells via ERK1/2 activation. Toxicology. 2007;230(1):76-82.

16. Li Y, Couch L, Higuchi M, et al. Mitochondrial dysfunction induced by sertraline, an antidepressant agent. Toxicol Sci. 2012;127(2):582-591.

17. Nikoletopoulou V, Markaki M, Palikaras K, et al. Crosstalk between apoptosis, necrosis and autophagy. Biochem Biophys Acta Mol Cell Res. 2013;1833(12):3448-3459.

18. Mignotte B, Vayssiere JL. Mitochondria and apoptosis. Eur J Biochem. 1998;252(1):1-15

19. Sánchez-Valle V, Chávez-Tapia NC, Uribe M, et al. Role of oxidative stress and molecular changes in liver fibrosis:a review. Curr Med Chem. 2012;19(28):4850-4860.

20. Higgins GC, Beart PM, Shin YS, et al. Oxidative stress:emerging mitochondrial and cellular themes and variations in neuronal injury. $J$ Alzheimers Dis. 2010;20(Suppl 2):S453-S473.

21. Gunawan BK, Kaplowitz N. Mechanisms of drug-induced liver disease. Clin Liver Dis. 2007;11(3):459-475.

22. Han D, Shinohara M, Ybanez MD, et al. Signal transduction pathways involved in drug-induced liver injury. Handb Exp Pharmacol. 2010;196:267-310.

23. Chen S, Melchior WB Jr, Guo L. Endoplasmic reticulum stress in drugand environmental toxicant-induced liver toxicity. J Environ Sci Health C Environ Carcinog Ecotoxicol Rev. 2014;32(1):83-104.

24. Henkel A, Green RM. The unfolded protein response in fatty liver disease. Semin Liver Dis. 2013;33(04):321-329.

25. Hotamisligil GS. Endoplasmic reticulum stress and the inflammatory basis of metabolic disease. Cell. 2010;140(6):900-917.

26. Kraskiewicz H, FitzGerald U. InterfERing with endoplasmic reticulum stress. Trends Pharmacol Sci. 2012;33(2):53-63.

27. Hummasti S, Hotamisligil GS. Endoplasmic reticulum stress and inflammation in obesity and diabetes. Circ Res. 2010;107(5):579-591.

28. Nagy G, Szarka A. BGP-15 inhibits caspase-independent programmed cell death in acetaminophen-induced liver injury. Toxicol Appl Pharmacol. 2010;243(1):96-103.

29. Li Y, Couch L, Higuchi M, et al. Mitochondrial dysfunction induced bysertraline, an antidepressant agent. Toxicol Sci. 2012;127:582-591.

30. Schonthal AH. Endoplasmic Reticulum Stress:Its Role in Disease and Novel Prospects for Therapy. Scientifica (Cairo). 2012;2012:857516. 
31. Murphy MP. Modulating mitochondrial intracellular location as a redox signal. Sci Signal. 2012;5(242):pe39.

32. Teschke R, Schulze J. Suspected herbal hepatotoxicity:requirements for appropriate causality assessment by the US Pharmacopeia. Drug Saf. 2012;35(12):1091-1097.

33. Pessayre D, Fromenty B, Berson A. Central role of mitochondria in drug-induced liver injury. Drug Metab Rev. 2012;44(1):34-87.

34. Pessayre D, Mansouri A, Berson A, et al. Mitochondrial involvement in drug-induced liver injury. Handb Exp Pharmacol. 2010;196:311-365.

35. Begriche K, Massart J, Robin MA, et al. Drug-induced toxicity on mitochondria and lipid metabolism:mechanistic diversity and deleterious consequences for the liver. J Hepatol. 2011;54(4):773-794.

36. Chalasani NP, Hayashi PH, Bonkovsky HL, et al. On behalf of the Practice Parameters Committee of the American College of Gastroenterology. ACG Clinical Guideline:The Diagnosis and Management of Idiosyncratic Drug-Induced Liver Injury. $\mathrm{Am} \mathrm{J}$ Gastroenterol. 2014;109:950-966.

37. Hussaini SH, Farrington EA. Idiosyncratic drug-induced liver injury:an overview. Expert Opin Drug Saf. 2007;6(6):673-684.

38. Roth RA, Ganey PE. Intrinsic versus idiosyncratic drug-induced hepatotoxicity-two villains or one? $J$ Pharmacol Exp Ther. 2010;332(3):692-697.

39. Bjornsson ES, Bergmann OM, Bjornsson HK, et al. Incidence, presentation, and outcomes in patients with drug-induced liver injury in the general population of Iceland. Gastroenterology. 2013;144(7):14191425 .

40. Grattagliano I, Bonfrate L, Diogo CV, et al. Biochemical mechanisms in drug-induced liver injury:certainties and doubts. World J Gastroenterol. 2009;15(39):4865-4876.

41. Devarbhavi H. An Update on Drug-induced Liver Injury. $J$ Clin Exp Hepatol. 2012;57(2):247-259.

42. Hartleb M, Biernat L, Kochel A. Drug-induced liver damage — a threeyear study of patients from one gastroenterological department. Med Sci Monit. 2002;8(4):CR292-CR296.

43. Herrine SK. Liver Injury Caused by Drugs.

44. Food and Drug Agency (FDA). Guidance for Industry Drug-Induced Liver Injury: Premarketing Clinical Evaluation.

45. Raschi E, De Ponti F. Drug- and herb-induced liver injury:Progress, current challenges and emerging signals of post-marketing risk. World J Hepatol. 2015;7(13):1761-1771.

46. Weiler S, Merz M, Kullak-Ublick GA. Drug-induced liver injury:the dawn of biomarkers? F1000 Prime Rep. 2015;7:34.

47. Bunchorntavakul C, Reddy KR. Acetaminophen-related hepatotoxicity Clin Liver Dis. 2013;17(4):587-607.

48. Fontana RJ, Watkins PB, Bonkovsky HL. Drug-Induced Liver Injury Network (DILIN) prospective study:rationale, design and conduct. Drug Saf. 2009;32(1):55-68.

49. Urban TJ, Goldstein DB, Watkins PB. Genetic basis of susceptibility to drug-induced liver injury:what have we learned and where do we go from here? Pharmacogenetics. 2012;13(7):735-738.

50. Regev A, Seeff LB, Merz M. Causality assessment for suspected DILI during clinical phases of drug development. Drug Saf. 2013;37:47-56.

51. Lee WM. Drug-induced hepatotoxicity. N Engl J Med. 2003;349:474485.
52. Chang CY, Schiano TD. Review article:drug hepatotoxicity. Aliment Pharmacol Ther. 2007;25(10):1135-1151.

53. Yuan L, Kaplowitz N. Mechanisms of drug-induced liver injury. Clin Liver Dis. 2013;17(4):507-518.

54. United States National Library of Medicine. LiverTox. Clinical and Research Information on Drug Induced Liver Toxicity.

55. Monshi MM, Faulkner L, Gibson A, et al. Human leukocyte antigen (HLA)-B*57:01-restricted activation of drug-specific $\mathrm{T}$ cells provides the immunological basis for flucloxacillin-induced liver injury. Hepatology. 2013;57(2):727-739.

56. Kröger H, Ehrlich W, Klewer M, et al. The influence of antagonists of poly (ADP-ribose) metabolism on acetaminophen hepatotoxicity. Gen Pharmacol. 1996;27(1):167-170.

57. Laster J, Satoskar R. Aspirin-Induced Acute Liver Injury. ACG Case Rep J. 2014;2(1):48-49.

58. Wallack EM, Callon R. Liver injury associated with the beta-interferons for MS. Neurology. 2004;63(6):1142-1143.

59. Raschi E, Poluzzi E, Koci A, et al. Liver injury with novel oral anticoagulants:assessing post-marketing reports in the US Food and Drug Administration adverse event reporting system. $\mathrm{Br} J$ Clin Pharmacol. 2015;80(2):285-293.

60. Hannaford PC, Kay CR, Vessey MP, et al. Combined oral contraceptives and liver disease. Contraception. 1997;55(3):145-151.

61. van Basten JP, van Hoek B, Zeijen R, et al. Glyburide-induced cholestatic hepatitis and liver failure. Case-report and review of the literature. Neth J Med. 1992;40(5-6):305-357.

62. Yan JY, Nie XL, Tao QM, et al. Ketoconazole associated hepatotoxicity: a systematic review and meta- analysis. Biomed Environ Sci. 2013;26(7):605-610.

63. Greenblatt HK, Greenblatt DJ. Liver injury associated with ketoconazole:review of the published evidence. J Clin Pharmacol. 2014;54(12):1321-1329.

64. de Denus S, Spinler SA, Miller K, et al. Statins and liver toxicity:a meta-analysis. Pharmacotherap. 2004; 24(5):584-591.

65. Tolman KG. The liver and lovastatin. Am J Cardiol. 2002;89(12):1374 1380.

66. Heidari R, Niknahad H, Jamshidzadeh A, et al. Factors affecting druginduced liver injury:antithyroid drugs as instances. Clin Mol Hepatol. 2014;20(3):237-248.

67. Roberts SM, DeMott RP, James RC. Adrenergic modulation of hepatotoxicity. Drug Metab Rev. 1997;29(1-2):329-353.

68. Manceaux P, Constant E, Zdanowicz N, et al. Management of marked liver enzyme increase during olanzapine treatment:a case report and review of the literature. Psychiatr Danub. 2011;23(Suppl 1):S15-S17.

69. Devarbhavi H, Andrade RJ. Drug-induced liver injury due to antimicrobials, central nervous system agents, and nonsteroidal antiinflammatory drugs. Semin Liver Dis. 2014;34(2):145-161.

70. Björnsson E. Hepatotoxicity associated with antiepileptic drugs. Acta Neurol Scand. 2008;118(5):281-290.

71. McLane J. Analysis of common side effects of isotretinoin. $J$ Am Acad Dermatol. 2001;45(5):S188-S194.

72. Durand F, Jebrak G, Pessayre D, et al. Hepatotoxicity of antitubercular treatments. Rationale for monitoring liver status. Drug Saf. 1996;15(6):394-405. 
73. Ko MS, Choi YH, Jung SH, et al. Tacrolimus therapy causes hepatotoxicity in patients with a history of liver disease. Int $J$ Clin Pharmacol Ther. 2015;53(5):363-371.

74. Stewart JD, Horvath R, Baruffini E, et al. Polymerase $\gamma$ gene POLG determines the risk of sodium valproate-induced liver toxicity. Hepatology. 2010;52(5):1791-1796.

75. Douglas IJ, Langham J, Bhaskaran K, et al. Orlistat and the risk of acute liver injury:self controlled case series study in UK Clinical Practice Research Datalink. BMJ. 2013;346:f1936.
76. Wilding J. Orlistat:should we worry about liver inflammation? BMJ. 2013;346:f2777.

77. Wooltorton E. Asthma drug zafirlukast (Accolate):serious hepatic events. CMAJ. 2004;170(11):1668.

78. Su CW, Wu JC, Huang YH, et al. Zafirlukast-induced acute hepatitis Zhonghua Yi Xue Za Zhi (Taipei). 2002;65(11):553-556. 